\title{
Stability of travelling waves in models of superconductivity
}

\author{
S. J. Chapman \\ Mathematical Institute, 24-29 St Giles', Oxford OX1 3LB, UK
}

[Received 7 February 1994 and in revised form 27 September 1994]

\begin{abstract}
The linear stability of two travelling waves describing phase changes in superconducting materials is examined. The first describes the change of phase between normally conducting and superconducting states in a type I superconductor, while the second describes the transition from the superconducting state to the mixed state in a type II superconductor.
\end{abstract}

\section{Introduction}

This paper is concerned with the stability of various phase changes in superconducting materials.

The response of a superconducting material to an applied magnetic field is conveniently described by Fig. 1, which shows the preferred state of the superconductor as a function of $H_{0}$, the applied magnetic field, and $\kappa$, a material parameter (known as the Ginzburg-Landau parameter) which determines the type of superconducting material: $\kappa<1 / \sqrt{ } 2$ describes what is known as a type I superconductor, $\kappa>1 / \sqrt{ } 2$ describes what is known as a type II superconductor.

For type I superconductors there is a critical magnetic field $H_{\mathrm{c}}$ (known as the thermodynamic critical field) below which the material will be in the superconducting state, but above which it will revert to the normally conducting (normal) state. The transition between normal and superconducting states as the applied magnetic field is raised or lowered through $H_{c}$ takes place by means of phase boundaries separating normal regions from superconducting regions sweeping through the material. In [3] and [7] the following free-boundary problem for this change of phase was derived:

$$
\begin{aligned}
\nabla^{2} \boldsymbol{H} & =\frac{\partial \boldsymbol{H}}{\partial t} & & \text { in } \Omega_{\mathrm{n}}, \\
\boldsymbol{H} & =\mathbf{0} & & \text { in } \Omega_{\mathrm{s}}, \\
|\boldsymbol{H}| & =H_{\mathrm{c}} & & \text { on } \Gamma, \\
\operatorname{curl} \boldsymbol{H} \wedge \boldsymbol{n} & =-v_{n} \boldsymbol{H} & & \text { on } \Gamma,
\end{aligned}
$$

where $\boldsymbol{H}$ is the magnetic field, $\Omega_{\mathrm{n}}$ is the normal region, $\Omega_{\mathrm{s}}$ is the superconducting region, $\Gamma$ is the free boundary separating these two regions (approached from the normal region), $n$ is the unit normal to $\Gamma$ (directed towards the superconducting region), and $v_{\mathrm{n}}$ is the normal velocity of $\Gamma$.

Experimental evidence $[9,10]$ seems to indicate that the switch-off of a superconducting wire (i.e. the transition from superconducting to normal) is a 


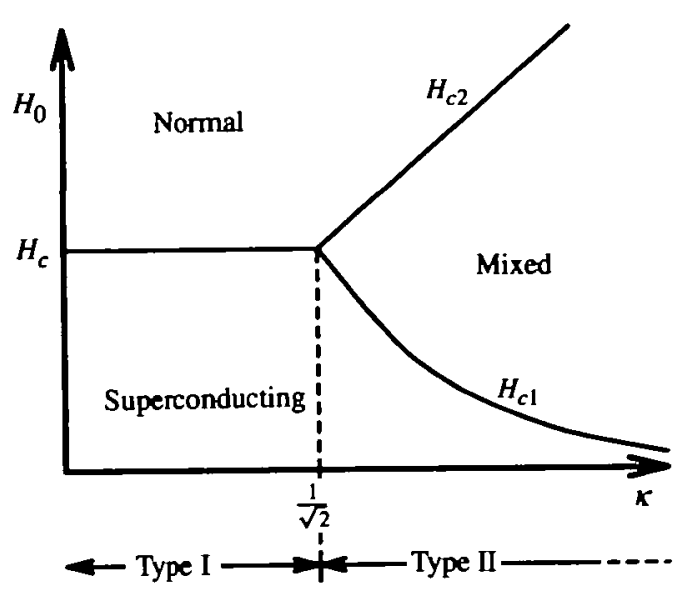

FIG. 1. The response of a superconducting material as a function of the applied magnetic field $H_{0}$ and the Ginzburg-Landau parameter $\kappa$.

stable process, while the switch-on (i.e. the transition from normal to superconducting) is unstable, with the appearance of fingers of superconducting material. Locally a smooth interface between normal and superconducting regions will be planar, so we hope to gain insight into the stability of an arbitrary interface by considering the stability of a planar one.

A solution of (1-4) representing a plane wave travelling with constant velocity is given by

$$
\boldsymbol{H}= \begin{cases}\left(0, H_{\mathrm{c}} \mathrm{e}^{-c(x-c t)}, 0\right) & \text { if } x<c t \\ 0 & \text { if } x>c t\end{cases}
$$

where the free boundary is given by $x=c t$, the normal region by $x<c t$, and the superconducting region by $x>c t$. In the next section we consider the linear stability of this solution.

For type II superconductors a third state exists, which is known as the mixed state. The mixed state, as its name suggests, is neither wholly superconducting nor wholly normal, but consists of many normal filaments embedded in a superconducting matrix. Each of these filaments carries with it a quantized amount of magnetic flux, and is circled by a vortex of superconducting current (thus these filaments are often known as vortices).

The transition from the normal state to the mixed state takes place via a bifurcation as the magnetic field is lowered through some critical value $H_{\mathrm{c} 2}$ (known as the upper critical field), and is described in $[1,2,4,11,12,14]$. This bifurcation is subcritical for type I superconductors, but supercritical for type II superconductors; hence the observation of the mixed state only for type II superconductors.

The magnetic field at which the superconducting state begins its transition to 
the mixed state has been studied in $[5]$. In $[6,8]$ a model was derived to describe the mixed state of a superconductor in which the vortices are averaged to give a vortex density, or vorticity. The change of phase from the superconducting state to the mixed state as vortices sweep through the sample can also be described by this model. The following equations were obtained-in two dimensions, with $\boldsymbol{H}=(0,0, H(x, y, t))$ :

$$
\begin{aligned}
\omega_{\mathrm{t}} & =\nabla \cdot(|\omega| \nabla H) \quad \text { in } \Omega_{\mathrm{m}}, \\
H-\nabla^{2} H & =\omega \quad \text { in } \Omega_{\mathrm{m}}, \\
\omega & =0 \quad \text { in } \Omega_{\mathrm{s}}, \\
H-\nabla^{2} H & =0 \quad \text { in } \Omega_{\mathrm{s}}, \\
{[H] } & =0, \\
{\left[\frac{\partial H}{\partial n}\right] } & =0, \\
v_{\mathrm{n}} & =-(\operatorname{sgn} \omega) \frac{\partial H}{\partial n} .
\end{aligned}
$$

Here $\Omega_{\mathrm{m}}$ is the mixed region, i.e. the region occupied by the vortices, and, as before, $\Omega_{3}$ is the superconducting region, $\Gamma$ is the free boundary separating these two regions, $n$ is the unit normal to $\Gamma$ (directed toward the superconducting region), $v_{\mathrm{n}}$ is the normal velocity of $\Gamma$ and [] stands for the jump in the enclosed quantity across $\Gamma ; H$ is the (now scalar) magnetic field, and $\omega$ is the vorticity.

Recent experiments [16] indicate that, in the presence of strong vortex pinning, when a wave of positive vorticity sweeps though a sample originally full of negative vorticity, the interface between the two regions is unstable and prone to fingering. With weaker pinning (obtained by increasing the temperature), the fingering seems to cease.

The model above describes the limit of no vortex pinning. Questions of interest are whether vortex fronts are stable in the absence of pinning, whether the instability depends on the sample previously having been full of vortices of the opposite sign, and whether the fingering is due to inhomogeneities in the underlying pinning forces, or is in fact due to a morphological instability of the interface. Here we will attempt to answer the first of these questions, by considering the stability of an interface between superconducting and vortex states in the clean (i.e. no pinning) limit. (We note that the model (6-12) can be modified to incorporate the effects of vortex pinning, and we hope to investigate questions of stability in this case in a later paper.)

Again, locally a smooth interface will be planar, so that we can gain insight into the stability of an arbitrary interface by considering the stability of a planar one. A solution of (6-12) representing a plane wave travelling with constant velocity is 
given by

$$
\begin{gathered}
\omega= \begin{cases}c-c(x-c t) & \text { if } x<c t, \\
0 & \text { if } x>c t,\end{cases} \\
H= \begin{cases}c-c(x-c t) & \text { if } x<c t, \\
c e^{-(x-c t)} & \text { if } x>c t,\end{cases}
\end{gathered}
$$

where the free boundary is given by $x=c t$, the vortex region is $x<c t$, and the superconducting region is $x>c t$. Note that in this case $c$ is required to be positive in order to satisfy equation $(12)$, so that $(13,14)$ can only describe the transition from the superconducting to the mixed state and not vice versa.

\section{Linear stability}

\subsection{Type I superconductors}

We perform a linear stability analysis of the solution (5) by considering perturbations to the boundary, which is given by $x=s$, of the form

$$
s(y, z, t)=c t+\epsilon \mathrm{e}^{\sigma t} \cos m y \cos n z,
$$

where $0<\epsilon \ll 1$, and $m$ and $n$ are real and positive for definiteness. The analysis is similar to that performed on the Stefan model in $[13,15]$. Indeed, when $m=0$, the two problems are identical since (1-4) reduces to a one-phase Stefan model in the cylindrical case in which $\boldsymbol{H}=(0, H(x, z, t), 0)$. We will see that the vectorial nature of the present problem leads to a decrease in instability for $m 0$, that is, the growth rate of unstable modes decreases with increasing $m$.

We expand the solution for $\boldsymbol{H}$ in powers of $\epsilon$ :

$$
\boldsymbol{H}=\left(0, H_{2}^{(0)}, 0\right)+\epsilon \boldsymbol{H}^{(1)}+\cdots,
$$

where $H_{2}^{(0)}$ is the unperturbed solution given by (5). Substituting the expansion $(16)$ into equation (1) and equating coefficients of $\epsilon$ yields

$$
\nabla^{2} \boldsymbol{H}^{(1)}=\frac{\partial \boldsymbol{H}^{(1)}}{\partial t}
$$

We will shortly change to coordinates moving with the free boundary, but we first calculate the boundary conditions for $\boldsymbol{H}^{(1)}$. We have

$$
\begin{aligned}
|\boldsymbol{H}(s, y, z)|^{2} & =H_{c}^{2}\left(1-2 \epsilon c e^{\sigma t} \cos m y \cos n z\right)+2 \epsilon H_{\mathrm{c}} H_{2}^{(1)}(c t, y, z)+O\left(\epsilon^{2}\right) \\
& =H_{\mathrm{c}}^{2}
\end{aligned}
$$

by (3). Equating coefficients of $\epsilon$ gives

$$
H_{2}^{(1)}(c t, y, z)=c H_{c} \mathrm{e}^{\sigma t} \cos m y \cos n z .
$$


We have

$v_{\mathrm{n}} H(s, y, z)=$

$$
-\left[\begin{array}{c}
\epsilon c H_{1}^{(1)}(c t, y, z) \\
c H_{c}+\epsilon\left(\sigma-c^{2}\right) H_{c} e^{v t} \cos m y \cos n z+\epsilon c H_{2}^{(1)}(c t, y, z) \\
\epsilon c H_{3}^{(1)}(c t, y, z)
\end{array}\right]^{\top}+O\left(\epsilon^{2}\right)
$$

while

$(\operatorname{curl} H \wedge n)(s, y, z)=$

$$
-\left[\begin{array}{c}
\epsilon c H_{\mathrm{c}} m \mathrm{e}^{\sigma t} \sin m y \cos n z \\
-c H_{c}+\epsilon\left(c^{2} H_{c} \mathrm{e}^{\sigma \tau} \cos m y \cos n z+\frac{\partial H_{2}^{(1)}}{\partial x}(c t, y, z)-\frac{\partial H_{1}^{(1)}}{\partial y}(c t, y, z)\right) \\
\epsilon\left(\frac{\partial H_{1}^{(1)}}{\partial z}(c t, y, z)-\frac{\partial H_{3}^{(1)}}{\partial x}(c t, y, z)\right)
\end{array}\right]^{\top}+O\left(\epsilon^{2}\right)
$$

Equating powers of $\epsilon$ in (4) therefore gives

$$
H_{1}^{(1)}(c t, y, z)=-m H_{c} \mathrm{e}^{\pi t} \sin m y \cos n z,
$$

$$
\begin{aligned}
& \frac{\partial H_{2}^{(1)}}{\partial x}(c t, y, z)-\frac{\partial H_{1}^{(1)}}{\partial y}(c t, y, z)=-c H_{2}^{(1)}(c t, y, z)-\sigma H_{\mathrm{c}} \mathrm{e}^{\sigma} \cos m y \cos n z, \\
& \frac{\partial H_{1}^{(1)}}{\partial z}(c t, y, z)-\frac{\partial H_{3}^{(1)}}{\partial x}(c t, y, z)=c H_{3}^{(1)}(c t, y, z) .
\end{aligned}
$$

Thus the problem for $\boldsymbol{H}^{(1)}$ is

$$
\nabla^{2} \boldsymbol{H}^{(1)}=\frac{\partial \boldsymbol{H}^{(1)}}{\partial t},
$$

with the (fixed) boundary conditions

$$
\begin{aligned}
H_{1}^{(1)}(c t, y, z) & =-m H_{c} \mathrm{e}^{\sigma t} \sin m y \cos n z, \\
H_{2}^{(1)}(c t, y, z) & =c H_{\mathrm{c}} \mathrm{e}^{\sigma t} \cos m y \cos n z, \\
\frac{\partial H_{2}^{(1)}}{\partial x}(c t, y, z) & =-\left(c^{2}+\sigma+m^{2}\right) H_{c} \mathrm{e}^{\sigma t} \cos m y \cos n z, \\
\frac{\partial H_{3}^{(1)}}{\partial x}(c t, y, z) & =-c H_{3}^{(1)}(c t, y, z)+m n H_{c} \mathrm{e}^{\sigma r} \sin m y \sin n z .
\end{aligned}
$$

We look for a solution for $H_{2}^{(1)}$ of the form

$$
H_{2}^{(1)}=F(x-c t) \mathrm{e}^{\sigma t} \cos m y \cos n z .
$$

Letting $\eta=x-c t$ and primes denote $d / d \eta$, we have

$$
\begin{gathered}
F^{\prime \prime}+c F^{\prime}-\left(n^{2}+m^{2}+\sigma\right) F=0, \\
F(0)=c H_{c} \\
F^{\prime}(0)=-\left(c^{2}+\sigma+m^{2}\right) H_{c}
\end{gathered}
$$

Hence

$$
F=A \mathrm{e}^{\lambda_{1} \eta}+B \mathrm{e}^{\lambda_{2} \eta}
$$


where $\lambda_{1}$ and $\lambda_{2}$ are the roots of

namely,

$$
\lambda^{2}+c \lambda-\left(n^{2}+m^{2}+\sigma\right)=0
$$

$$
\lambda_{1}=\frac{-c+\left[c^{2}+4\left(n^{2}+m^{2}+\sigma\right)\right]^{\frac{1}{2}}}{2} \text { and } \lambda_{2}=\frac{-c-\left[c^{2}+4\left(n^{2}+m^{2}+\sigma\right)\right]^{\frac{1}{2}}}{2} \text {. }
$$

The boundary conditions $(29,30)$ imply

$$
\begin{aligned}
A+B & =c H_{c}, \\
A \lambda_{1}+B \lambda_{2} & =-\left(c^{2}+\sigma+m^{2}\right) H_{c} .
\end{aligned}
$$

We consider separately the cases $c>0$ and $c<0$.

(1) $c>0$. If we require that $H_{2}^{(1)}$ should be small compared to $H_{2}^{(0)}=H_{c} \mathrm{e}^{-c(x-a)}$ as $x-c t \rightarrow-\infty$, then we have either $\operatorname{Re}\left\{\lambda_{2}\right\}>-c$ or $B=0$. In the first case the solution would grow exponentially as $x-c t \rightarrow-\infty$, but would still be small compared to the leading-order solution. In particular, if we were treating the problem as an initial value problem such a solution would correspond to an initial perturbation which grows exponentially at infinity, while the eigenmodal case $B=0$ corresponds to an initial perturbation which decays at infinity. Of course, a general initial perturbation will be a sum involving both types.

If $\operatorname{Re}\left\{\lambda_{2}\right\}>-c$, then

that is,

$$
\operatorname{Re}\left\{c^{2}+4\left(n^{2}+m^{2}+\sigma\right)\right\}<\left(\operatorname{Re}\left\{\left[c^{2}+4\left(n^{2}+m^{2}+\sigma\right)\right]^{\frac{1}{2}}\right\}\right)^{2}<c^{2},
$$

$$
\operatorname{Re}\{\sigma\}<-\left(n^{2}+m^{2}\right) \text {. }
$$

Hence $\operatorname{Re}\{\sigma\}$ is negative. If we have $B=0$, then (31) implies $A=c H_{c}$, which in (32) implies

$$
c\left[c^{2}+4\left(n^{2}+m^{2}+\sigma\right)\right]^{\frac{1}{2}}=-c^{2}-2 \sigma-2 m^{2} .
$$

Squaring gives

$$
c^{4}+4 c^{2}\left(n^{2}+m^{2}+\sigma\right)=c^{4}+4 \sigma^{2}+4 m^{4}+4 c^{2} \sigma+4 c^{2} m^{2}+8 \sigma m^{2},
$$

that is,

Hence

$$
c^{2} n^{2}=\left(\sigma+m^{2}\right)^{2}
$$

$$
\sigma=-m^{2} \pm n c
$$

However, since we had to square our equation to obtain this answer, we need to substitute (34) into (33) and check for consistency. We find

$$
c\left[(c \pm 2 n)^{2}\right]^{\frac{1}{2}}=-c(c \pm 2 n),
$$

that is, $c \pm 2 n<0$. Since $c>0$ we must have

$$
\sigma=-m^{2}-n c \text { and } 2 n>c .
$$

Since $c$ is positive, we see that $\sigma$ is negative and the solution is linearly stable.

(2) $c<0$. If we again require that $H_{2}^{(1)}$ should be small compared to $H_{2}^{(0)}=$ $H_{c} \mathrm{e}^{-c(x-a)}$ as $x-c t \rightarrow-\infty$, then we must have $B=0$. As before $\sigma=-m^{2} \pm n c$, 
with $c \pm 2 n<0$. Thus

$$
\sigma= \begin{cases}-m^{2} \pm n c & \text { if } 2 n<-c \\ -m^{2}-n c & \text { if } 2 n>-c\end{cases}
$$

Hence, for $n / m^{2}>-c$ there will be a solution with $\sigma>0$ indicating an instability of the boundary.

We note the very different responses to perturbations in the $y$ and $z$ directions. We see that for a perturbation in the $y$-direction only $(n=0)$ the boundary is always stable. However, for a perturbation in the $z$-direction only $(m=0)$ the boundary is stable if and only if $c>0$, i.e. the normal region is advancing. Furthermore, when both perturbations are present, the perturbation in the $y$-direction serves to increase the stability of the perturbation in the $z$-direction when $c>0$, and decrease the instability of the perturbation in the $z$-direction when $c<0$.

In (1) we found that no mode solutions decaying at infinity were possible when $2 n<c$ (and yet such a perturbation may be applied to the system and it will evolve in time). A complete linear treatment for a given perturbation can be obtained by taking the Laplace transform in time, i.e. treating the problem as an initial value problem. If this approach is carried out, we find that the free boundary is of the form

$$
x=c t+\frac{\cos m y \cos n z}{2 \pi \mathrm{i}} \int_{\gamma-\mathrm{ix}}^{\gamma+\mathrm{i} \alpha} \hat{\sigma} \mathrm{e}^{p t} \mathrm{~d} p
$$

where the integrand $\hat{\sigma}$ has poles at the roots of the dispersion relation (33). There is also a branch cut in the $p$ plane from $-\frac{1}{4} c^{2}-n^{2}-m^{2}$ to $-\infty$. When $2 n<c$, the sole contribution to the integrand comes from this branch cut. Since $\operatorname{Re}\{p\}>0$ there, this contribution decays with time.

Following the same reasoning as for the Stefan problem, the instability of perturbations in the $z$-direction in the case $c>0$ can be explained physically by considering the effects of a small 'bump' on the boundary in the two-dimensional case when $\boldsymbol{H}=(0, H(x, z, t), 0)$. When the normal region is expanding, such a bump will serve to lower locally the gradient of the magnetic field and hence reduce the velocity of the boundary in the vicinity of the bump, leading to stability. However, when the normal region is contracting, such a bump will increase locally the gradient of the magnetic field and hence increase the velocity of the boundary, leading to instability.

In contrast to perturbations in the $z$-direction, perturbations in the $y$-direction require the bending of lines of magnetic field, since the magnetic field must always be parallel to the interface. Such bending raises the energy associated with the magnetic field, and it is this which causes perturbations in the $y$-direction to decay.

Finally, we consider which modes, i.e. which values of $\sigma$, will actually be observed in practice. In the case $c<0$ the most unstable mode corresponds to $(m, n)=(0, \infty)$. Thus the modes observed will correspond to two-dimensional fingers (uniform in the $y$-direction). In the present model the wavelength of the 
fingering would be zero. Adding a term proportional to curvature to equation (3), corresponding to the surface energy associated with a normal/superconducting domain boundary (see [3]), would presumably stabilize the very short wavelengths, and hence select the wavelength of the fingering.

In the stable case $c>0$ the modes which have the smallest decay rate are those corresponding to small values of $m$ and $n$. Hence long-wavelength perturbations will persist for a greater time than short-wavelength perturbations.

\subsection{Type II superconductors}

We perform a similar analysis of the solution $(13,14)$ by considering perturbations to the boundary of the form

$$
s=c t+\epsilon \mathrm{e}^{\sigma t} \cos k y .
$$

We expand the corresponding solution $(\omega, H)$ in powers of $\epsilon$ :

$$
\begin{gathered}
\omega=\omega_{0}+\epsilon \omega_{1}+\cdots, \quad H=H_{0}+\epsilon H_{1}+\cdots, \quad \text { for } x<s, \\
\omega=0, \quad H=H_{0}+\epsilon H_{1}+\cdots, \quad \text { for } x>s,
\end{gathered}
$$

where $\left(\omega_{0}, H_{0}\right)$ is the unperturbed solution given by $(13,14)$. Substituting these expansions into (6-9) and equating coefficients of $\epsilon$ yields

$$
\begin{aligned}
& \frac{\partial \omega_{1}}{\partial t}=\nabla \cdot\left(\omega_{0} \nabla H_{1}+\omega_{1} \nabla H_{0}\right) \quad \text { for } x<s, \\
& H_{1}-\nabla^{2} H_{1}=\omega_{1} \quad \text { for } x<s, \\
& H_{1}-\nabla^{2} H_{1}=0 \quad \text { for } x>s .
\end{aligned}
$$

As before, we will shortly change to coordinates moving with the free boundary, but we first calculate the boundary conditions on (40-42).

We have

$$
H(s, y, t)=H_{0}(c t, y, t)+\epsilon\left(H_{1}(c t, y, t)-\frac{\partial H_{0}}{\partial x}(c t, y, t) \mathrm{e}^{\sigma} \cos k y\right)+O\left(\epsilon^{2}\right) .
$$

Equating coefficients of $\epsilon$ in (10) we find

$$
\left[H_{1}\right]_{x=a^{-}}^{x-a+}=-\mathrm{e}^{\sigma x} \cos k y\left[\frac{\partial H_{0}}{\partial x}\right]_{x=c^{-}}^{x=a+}=0
$$

since $\partial H_{0} / \partial x$ is continuous across $\Gamma$. Similarly, equating powers of $\epsilon$ in (11) yields

$$
\left[\frac{\partial H_{1}}{\partial x}\right]_{x=c t-}^{x=c t+}=-\mathrm{e}^{\sigma t} \cos k y\left[\frac{\partial^{2} H_{0}}{\partial x^{2}}\right]_{x-c t-}^{x=a+}=-c \mathrm{e}^{o t} \cos k y .
$$

Finally, since the normal velocity of the interface is given by

$$
v_{\mathrm{n}}=c+\epsilon \sigma \mathrm{e}^{\sigma t} \cos k y+O\left(\epsilon^{2}\right)
$$


equating coefficients of $\epsilon$ in (12) gives

$$
\frac{\partial H_{1}}{\partial x}(c t-, y, t)=-\sigma \mathrm{e}^{\sigma t} \cos k y .
$$

Thus the problem we have is given by (40-42) with boundary conditions (44-46). We seek a solution of the form

$$
\begin{aligned}
& \omega_{1}=\mathrm{e}^{\sigma t} \cos k y u(\eta), \\
& H_{1}=\mathrm{e}^{\sigma r} \cos k y v(\eta),
\end{aligned}
$$

where $\eta=x-c t$. Then

$$
\begin{aligned}
\sigma u & =-c v^{\prime}+c(1-\eta)\left(v^{n}-k^{2} v\right) \quad(\eta<0), \\
v^{n}-\left(1+k^{2}\right) v & =-u \quad(\eta<0), \\
v^{n}-\left(1+k^{2}\right) v & =0 \quad(\eta>0), \\
{[v]_{0^{+}}^{0+} } & =0, \\
{\left[v^{\prime}\right]_{0^{+}}^{0+} } & =-c, \\
v^{\prime}\left(0^{-}\right) & =-\sigma,
\end{aligned}
$$

where primes denote $d / d \eta$. The solution of equation (51) which decays at infinity is

$$
v=a \mathrm{e}^{-\left(1+k^{2}\right)^{1 / \eta} \eta} \quad(\eta>0)
$$

Since $v^{\prime}(0+)=-\sigma-c$ we have that $a=(\sigma+c) /\left(1+k^{2}\right)^{\frac{1}{2}}$. Eliminating $u$ we therefore have

$$
\begin{aligned}
(\sigma+c-c \eta) v^{\prime \prime}-c v^{\prime}+\left(c k^{2} \eta-\left(1+k^{2}\right) \sigma-c k^{2}\right) v=0 \quad(\eta<0), \\
v(0)=\frac{\sigma+c}{\left(1+k^{2}\right)^{\frac{1}{2}}} \\
v^{\prime}(0)=-\sigma \\
v^{\prime} \rightarrow 0 \quad \text { as } \eta \rightarrow-\infty .
\end{aligned}
$$

We prove that $\sigma \leqslant 0$. Suppose not; then $v(0)>0$ and $v^{\prime}(0)<0$, while (56) and (59) imply that $v \rightarrow 0$ as $\eta \rightarrow-\infty$. Since (56) implies that $v$ cannot have a positive maximum, $v$ cannot satisfy this boundary condition at $-\infty$, and we have a contradiction. Thus the interface is linearly stable.

The transformation $v=\mathrm{e}^{-\frac{1}{b}} f(\zeta)$, with $\zeta=2 k(c \eta-c-\sigma)$, converts (56) into Kummer's equation, from which we can obtain a (rather ungainly) dispersion relation in terms of Whittaker functions. This dispersion relation is shown in Fig. 


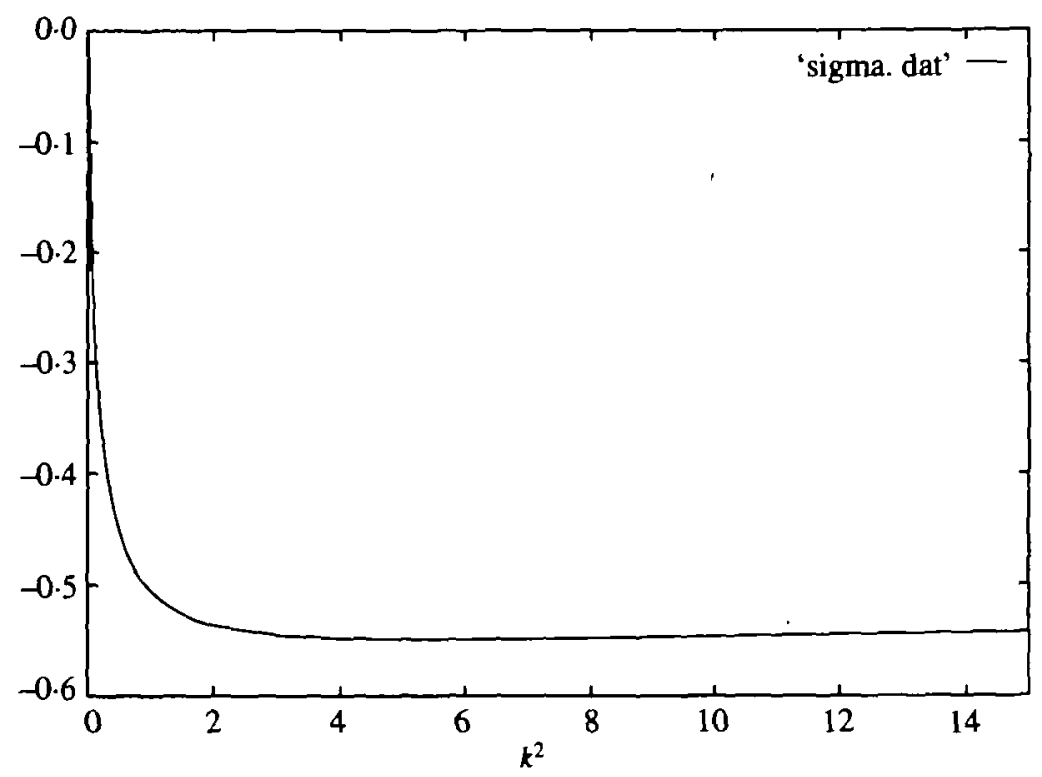

Fig. 2. The dispersion relation, showing $\sigma / c$ as a function of $k^{2}$.

2, which is a graph of $\sigma / c$ against $k^{2}$. We see that for $k^{2}$ greater than about $2, \sigma$ is roughly constant. In fact $\sigma$ asymptotes to $-\frac{1}{2} c$ as $k \rightarrow \infty$, as we now show.

For large $k$, we rescale by setting $\xi=k \eta$ and $g(\xi)=k v(\eta)$, to obtain

$$
\begin{aligned}
\left(\sigma+c-\frac{c \xi}{k}\right) g^{\prime \prime}-\frac{c}{k} g^{\prime}+\left[\frac{c \xi}{k}-\left(1+\frac{1}{k^{2}}\right) \sigma-c\right] g=0 \quad(\xi<0), \\
g(0)=\frac{(\sigma+c) k}{\left(1+k^{2}\right)^{\frac{1}{2}}} \\
g^{\prime}(0)=-\sigma, \\
g^{\prime} \rightarrow 0 \text { as } \xi \rightarrow-\infty
\end{aligned}
$$

The leading-order behaviour as $k \rightarrow \infty$ is given by

$$
\begin{aligned}
& g_{0}^{n}=g_{0}, \\
& g_{0}(0)=\sigma_{0}+c, \\
& g_{0}^{\prime}(0)=-\sigma_{0}, \\
& g_{0}^{\prime} \rightarrow 0 \text { as } \xi \rightarrow-\infty .
\end{aligned}
$$

Hence $g=\left(\sigma_{0}+c\right) \mathrm{e}^{\xi}$ and $\sigma_{0}=-\frac{1}{2} c$. 


\section{Conclusions}

We have examined the linear stability of planar travelling waves in two models for phase changes in superconducting materials.

The first was a model for type I superconductors, with the change of phase being between normal and superconducting states. We found that a plane wave was stable if the normal region was expanding, but unstable if the superconducting region was expanding.

The second model described the change of phase from the superconducting state to the mixed state in a type $I$ superconductor. Here we found that a plane wave was stable to small perturbations.

Both these results agree qualitatively with experimental observations $[9,10,16]$. In a future paper we hope to extend the analysis of the mixed state/superconducting state transition to include the effects of vortex pinning. In this case experimental observations indicate that the interface is prone to fingering [16], but it is unclear whether this is caused by a morphological instability or by inhomogeneity in the underlying distribution of pinning sites.

\section{REFERENCES}

1. Abrikosov, A. A., 1957. On the magnetic properties of superconductors of the second group. Sov. Phys. JETP 5, 1174-82.

2. BOlley, C., \& HelfFer, B., 1993. Rigorous results on Ginzburg-Landau models in a film submitted to an exterior parallel magnetic field. Preprint, Ecole centrale de Nantes.

3. Chapman, S. J., 1995. Asymptotic analysis of the Ginzburg-Landau model of superconductivity: Reduction to a free boundary model. $Q$. Appl. Math., to appear.

4. Chapman, S. J., 1994. Nucleation of superconductivity in decreasing fields I and II. Eur. J. Appl. Math. 5, 449-94.

5. Chapman, S. J., 1995. Superheating field of type II superconductors. SIAM J. Appl. Math., to appear.

6. Chapman, S. J., 1995. A three-dimensional mean-field model of superconducting vortices. SIAM J. Appl. Math., to appear.

7. Chapman, S. J., Howison, S. D., \& OCKendon, J. R., 1992. Macroscopic models of superconductivity. SIAM Rev. 34, 529-60.

8. Chapman, S. J., Rubinstein, J., \& Schatzman, M., 1995. A mean-field model of superconducting vortices. Eur. J. Appl. Math., to appear.

9. Faber, T. E., 1953. Phase transition in superconductors. II. Phase propagation above the critical field. Proc. R. Soc. Lond. A 219, 75-88.

10. FABER, T. E., 1954. Phase transition in superconductors. III. Phase propagation below the critical field. Proc. R. Soc. Lond. A 223, 174-94.

11. Kleiner, W. H., Roth, L. M., \& Autler, S. H., 1964. Bulk solution of GinzburgLandau equations for type II superconductors: Upper critical field region. Phys. Rev. A 133, 1226-7.

12. Millman, M. H., \& Keller, J. B., 1969. Perturbation theory of nonlinear boundaryvalue problems. J. Math. Phys. 10, 342.

13. Mullins, W. W., \& Sekerka, R. F., 1963. Morphological stability of a particle growing by diffusion or heat flow. J. Appl. Phys. 34, 323-9.

14. ODEH, F., 1967. Existence and bifurcation theorems for the Ginzburg-Landau equations. J. Math. Phys. 8, 2351-6.

15. Turland, B. D., \& Peckover, R. S., 1980. Stability of planar melting fronts in Stefan problems. J. Inst. Math. Applic. 25, 1-15.

16. Vlasko-Vlasov, V., 1993. Video presentation at the Workshop on the Statistics and Dynamics of Vortices in Superconductors, Eugene. 